\title{
DISTORÇÕES CAUSADAS PELA FALTA DE CALIBRAÇÃO RADIOMÉTRICA E EFEITOS ATMOSFÉRICOS NA ANÁLISE DE DADOS DE SENSORIAMENTO REMOTO ORBITAL
}

\author{
André WELTER ${ }^{1}$ \\ Thiago STATELLA ${ }^{2}$ \\ Geneci Marques de LIMA3
}

\begin{abstract}
Resumo
O artigo investiga distorções nas informações extraídas de dados de sensores remotos quando não são feitas calibração radiométrica e correção de efeitos atmosféricos. Foram comparados valores de pixels em unidades de Níveis Digitais, reflectância sem correção (reflectância no topo da atmosfera) e reflectância com correção (reflectância de superfície) de efeitos atmosféricos, obtidos de alvos específicos (área queimada, corpo d'água, cultura agrícola, vegetação nativa e solo exposto) de uma imagem multiespectral adquirida pelo satélite Landsat 7, órbita 226, ponto 071, sobre a região de Cuiabá - MT, do dia 28/09/2002. Em seguida, foram comparados índices de vegetação da diferença normalizada obtidos a partir dos dados não calibrados (ND) e reflectâncias de superfície (com correção) a fim de verificar o impacto da utilização dos dados brutos. A análise demonstra a importância de se considerar os efeitos atmosféricos nos estudos realizados com informações obtidas por sensores remotos orbitais (que, em muitos casos, não são corrigidas), uma vez que as assinaturas espectrais extraídas dos dados sem tratamento destoam significativamente do esperado, segundo a literatura especializada.
\end{abstract}

Palavras-chave: Detecção remota. Plataformas orbitais. Espalhamento atmosférico. Reflectância de superfície. Reflectância no topo da atmosfera.

\section{DISTORTIONS CAUSED BY THE LACK OF RADIOMETRIC CALIBRATION AND ATMOSPHERIC EFFECTS ON THE ANALYSIS OF ORBITAL REMOTE SENSING DATA}

\footnotetext{
Abstract

This paper investigates the distortions in information extracted from remote sensing data when radiometric calibration and atmospheric effects correction are not carried out. For the comparison, we used pixel values from raw data in Digital Numbers, from the top of the atmosphere reflectance (TOA) uncorrected

1 Serviço de Cartografia, Instituto Nacional de Colonização Agrária - INCRA. E-mail: andrelwgeo@gmail.com

2 Departamento de Área de Construção Civil, Instituto Federal de Educação, Ciência e Tecnologia de MT - IFMT. E-mail: thiago.statella@cba.ifmt.edu.br

${ }^{3}$ Departamento de Área de Construção Civil, Instituto Federal de Educação, Ciência e Tecnologia de MT - IFMT. E-mail: limageneci46@gmail.com
} 
data, and from the surface reflectance data (SR), gathered from selected targets (such as burned crop, native vegetation, rivers and bare soil) in a multispectral image acquired by the Landsat 7 satellite, row 226, path 071, depicting the Cuiabá city in MT state, on 28/09/2002. Next, we compared Normalized Difference Vegetation Index calculated from the uncalibrated data (DN) and from the surface reflectances (atmosphere corrected) so that we could verify the errors when using raw data. The analysis shows the importance of the atmospheric correction prior the analysis when dealing with orbital sensors (in several cases not corrected), given that the spectral signatures of the targets were severely drifted from the ones expected according to the specialized literature.

Keywords: Remote detection. Orbital platform. Atmospheric reflection. Surface reflectances. Top of the atmosphere reflectance.

\section{DISTORCIONES CAUSADAS POR FALTA DE CALIBRACIÓN RADIOMÉTRICA Y EFECTOS ATMOSFÉRICOS EN EL ANÁLISIS DE DATOS DE DETECCIÓN REMOTA ORBITAL}

\section{Resumen}

El artículo investiga las distorsiones en la información extraída de los datos del sensor remoto cuando no se realizan la calibración radiométrica y la corrección de los efectos atmosféricos. Los valores de píxeles se compararon en unidades de niveles digitales, reflectancia sin corrección (reflectancia en la parte superior de la atmósfera) y reflectancia con corrección (reflectancia superficial) de los efectos atmosféricos, obtenidos de objetos específicos (quemadas, cuerpo de agua, cultivo, vegetación nativa y suelo expuesto) de una imagen multiespectral adquirida por el satélite Landsat 7, órbita 226, punto 071, sobre la región de Cuiabá - MT, del 28/09/2002. Además, se compararon los índices de vegetación de diferencia normalizada obtenidos de los datos no calibrados (ND) y las reflectancias de la superficie (con corrección) para verificar el impacto del uso de datos sin procesar. El análisis demuestra la importancia de considerar los efectos atmosféricos en estudios realizados con información obtenida por sensores orbitales remotos (que, em muchos casos, no se corrigen), ya que las caracteristicas espectrales extraídas de los datos sin tratamiento difieren significativamente de lo esperado, según la literatura especializada.

Palabras clave: Detección remota. Plataformas orbitales. Dispersión atmosférica. Reflectancia superficial. Reflectancia en la parte superior de la atmósfera.

\section{INTRODUÇÃO}

Atualmente, os métodos de Sensoriamento Remoto tornaram-se uma ferramenta indispensável para diversas atividades práticas que estão inseridas no cotidiano das pessoas que vivem no meio urbano e rural (como monitoramento de culturas agrícolas, análise para regularização fundiária, 
mapas de localização e traçado de rotas, etc.). Dados de sensores remotos são utilizados para otimizar custos de aquisição de informações espectrais sobre grandes áreas, facilitar planejamento, monitoramento ambiental ou de produção, visando obter melhores resultados e maior controle sobre os processos. Contudo, quando não tratadas adequadamente, imagens de satélite fatalmente levarão o usuário a equívocos e análises incorretas.

As imagens de satélite resultam da leitura obtida, por sensores orbitais, da Radiação Eletromagnética (REM) refletida pela superfície terrestre (radiância). Esta energia, quando na região do espectro visível, sofre espalhamento e absorção pelos vários constituintes atmosféricos antes de chegar ao sensor. Os menores comprimentos de onda na região do visível sofrem espalhamento molecular (ou de Rayleigh), causado por moléculas de gás. Partículas de poeira e fumaça atenuam os maiores comprimentos de onda do espectro visível, num efeito conhecido como espalhamento Mie. Ainda, há o espalhamento não seletivo, causado por vapor d'água, e cuja magnitude independe do comprimento de onda da energia eletromagnética. Apesar disso, alguns trabalhos não apresentam a correção atmosférica em seus dados (ANDRADE et al., 2018; SILVIA et al., 2011), alguns aplicam métodos aproximados (LEITE et al., 2017), como o Dark Object Subtraction (que opera no contradomínio dos ND) e, ainda, há trabalhos em que a calibração radiométrica e correções atmosféricas não são aplicadas (RIBEIRO et al., 2018; ZANZARINI et al., 2013; VELASCO et al., 2007). Não raro, o contradomínio das imagens utilizadas é um intervalo de Níveis Digitais (ND, ou tons de cinza), quando deveria ser um intervalo de radiâncias (em $w^{-2} s r^{-1} \mu m^{-1}$ ) ou reflectâncias, que são grandezas com significado físico, diretamente correlacionadas às características físico-químicas dos alvos.

Este artigo tem o objetivo de demonstrar as distorções ocorridas nas análises de imagens quando a calibração radiométrica e efeitos atmosféricos são negligenciados. Isto é demonstrado graficamente e quantitativamente pela comparação entre valores de pixels em unidades de ND, reflectância no topo da atmosfera (TOA - Top of the Atmosphere) e reflectância de superfície (RS, ou seja, reflectância corrigida de efeitos atmosféricos), obtidos de alvos específicos (área queimada, corpo d'água, cultura agrícola, vegetação nativa e solo exposto), 
de uma imagem multiespectral adquirida pelo satélite Landsat 7 (órbita/ponto 226/071), sobre Cuiabá - MT e região, no dia 28/09/2002, período do ano de estiagem, sem a presença de nuvens na cena. Além disso, foram comparados índices de vegetação por diferença normalizada (Normalized Difference Vegetation Index - NDVI) obtidos a partir das reflectâncias no topo da atmosfera e de superfície a fim de verificar o impacto da utilização dos dados não corrigidos dos efeitos da atmosfera em vez dos dados tratados. Uma vez que toda cena adquirida por plataforma orbital possuirá distorções causadas pela atmosfera (a depender apenas do tipo de mecanismo espalhador em ação), tais efeitos são bem caracterizados neste caso de estudo, não havendo a necessidade de se processar um conjunto maior de imagens.

O trabalho está organizado da seguinte forma: no item 2 são abordados aspectos teóricos sobre a correção atmosférica de dados de sensores remotos, no item 3 apresenta-se a área de estudo, no item 4 são dados detalhes do método empregado para analisar as imagens, no item 5 são exibidos os resultados e, por fim, no item 6 faz-se a conclusão do trabalho, que é seguida do item de referências bibliográficas.

\section{CORREÇÃO DE EFEITOS ATMOSFÉRICOS}

Segundo Novo (2010), no Sensoriamento Remoto da superfície terrestre a principal fonte de radiação eletromagnética é o Sol. A radiação gerada pelas reações em seu interior se propaga na forma de ondas ou partículas em todas as direções e está sujeita à interação com a atmosfera e seus componentes. Os componentes da atmosfera que mais influenciam na absorção da radiação eletromagnética que efetivamente chega à superfície da Terra são: o vapor d'água $\left(\mathrm{H}_{2} \mathrm{O}\right)$, o Oxigênio $\left(\mathrm{O}_{2}\right)$, o Ozônio $\left(\mathrm{O}_{3}\right)$ e o Gás Carbônico $\left(\mathrm{CO}_{2}\right)$, que formam uma atmosfera opaca que barra parte radiação antes de ocorrer a interação com os objetos da superfície terrestre. Para Antunes et al. (2003) a reflectância obtida da mistura de sinal da atmosfera e da superfície é dita reflectância aparente ou reflectância no topo da atmosfera (TOA), sendo a reflectância definida como a razão entre o fluxo refletido pelo alvo e o fluxo incidente no mesmo (NOVO, 2010). 
A atmosfera absorve ou espalha a radiação eletromagnética de forma diferenciada em função dos seus comprimentos de onda. As partículas presentes na atmosfera, como aerossóis, poeiras, moléculas de diversos gases com diferentes tamanhos interferem na radiação que chega aos alvos terrestres ou ao sensor. Ou seja, a energia eletromagnética ao atingir a atmosfera é por esta espalhada, e parte desta energia espalhada retorna para o espaço, vindo a contaminar a energia refletida ou emitida pela superfície e que é detectada pelos sensores orbitais (PONZONI et al., 2015).

A diminuição do contraste entre objetos de uma mesma cena, a redução da possibilidade de identificação de pequenos objetos ou a diferenciação entre objetos de uma cena são consequência dos efeitos da atmosfera nas imagens obtidas por sensoriamento remoto, além da clara alteração na radiância medida pelo sensor.

Novo (2010) entende que a correção dos efeitos atmosféricos é importante especialmente em três casos específicos: quando se tem a necessidade de recuperar o valor da grandeza radiométrica medida, ou seja, quando se quer conhecer a reflectância, emitância ou retroespalhamento do objeto em estudo, para poder utilizar estes valores em modelos empíricos ou teóricos; quando se pretende utilizar algoritmos que se baseiam em operações aritméticas entre bandas, como o NDVI; ou quando se objetiva comparar imagens de diferentes datas em termos de propriedades dos objetos na cena.

A correção atmosférica é essencial para as análises de imagens de satélite, devido ao fato que a interação entre os gases e as partículas presentes na atmosfera com o fluxo de energia eletromagnética vinda do sol prejudica a passagem das ondas eletromagnéticas através da atmosfera, causando interferências. Essas interferências acontecem devido a três tipos de interações atmosféricas na energia reemitida por um alvo, quando este é visualizado do espaço. Estas alterações são conhecidas como espalhamento, absorção e reflectância (NOVO, 2010).

Existem três formas de espalhamentos: Rayleigh, Mie e não-seletivo. $\mathrm{O}$ espalhamento Rayleigh é produzido pelas partículas de gases constituintes da atmosfera cujos diâmetros são menores que o $\lambda$ (comprimento de onda) da radiação. O espalhamento Mie, provocado por fumaça e poeira, ocorre quando o 
diâmetro das partículas presentes na atmosfera é aproximadamente igual ao $\lambda$ da radiação. E o espalhamento não-seletivo, provocado por vapor d'água, ocorre quando o diâmetro das partículas espalhadoras é muito maior que os comprimentos de onda, neste caso a REM de diferentes comprimentos de onda será espalhada com igual intensidade. Todo espalhamento ocorre pela absorção e remissão aleatória de radiação por átomos ou moléculas presentes na atmosfera (JENSEN, 2009).

Vários métodos para correção destes efeitos são encontrados na literatura, desde os mais simples, de correção relativa, como o DOS (Dark Object Subtraction), cujo contradomínio são ND (e partem da premissa de que em toda cena existe um alvo com radiância nula, o que não se verifica em muitas situações), até os mais precisos, que utilizam algoritmos para modelagem da transferência radiativa da atmosfera, e que operam sobre radiância e/ou reflectância (CHAVEZ, 1988; MATTHEW et al., 2002; RUMORA et al., 2020). Estes últimos são conhecidos como de correção absoluta, e requererem a prévia calibração radiométrica dos dados brutos, processo pelo qual os valores de ND (quantização das radiâncias) são convertidos em radiância (valor com significado físico realmente medido pelo sensor). Isso pode ser feito a partir dos parâmetros de calibração radiométrica dos sensores, normalmente disponíveis para usuários no caso de sensores orbitais por meio de relatórios técnicos e/ou documentação fornecida pelo fabricante das câmeras.

Para as imagens do sensor ETM+ a bordo do Landsat 7, utilizado nesta pesquisa, é comum a adoção do modelo 6S (Second Simulation of the Satellite Signal in the Solar Spectrum), baseado no cálculo da transferência radiativa na atmosfera. Segundo Antunes et al. (2003) o modelo 6S é uma versão aprimorada do $5 \mathrm{~S}$ (Simulação do sinal de satélite no espectro solar), feito pelo Laboratoire d'Optique Atmospherique. O modelo $6 \mathrm{~S}$ foi desenvolvido por Vermote et al. (1997), para simular a radiância que chega nos sensores em bandas dentro do espectro solar, entre $0,25\lceil\mathrm{~m}$ a 4,00 $\{\mathrm{m}$. Sendo assim, pode-se calcular a reflectância de superfície (RS) do alvo, isto é, a reflectância livre dos efeitos de espalhamento atmosférico. 


\section{CENA DE ESTUDO}

A imagem multiespectral escolhida para o trabalho foi a adquirida pelo sensor ETM+ a bordo do satélite Landsat 7, órbita/ponto 226/071, na data de 28/09/2002, na região de Cuiabá - MT, nas bandas 1 (azul, o,45 - 0,52 (m), 2 (verde, o,52 - o,60 $(\mathrm{m}$ ), 3 (vermelho, o,63 - o,69 $\lceil\mathrm{m}$ ), 4 (infravermelho próximo, 0,76-0,90 $(\mathrm{m}$ ), 5 (infravermelho médio, 1,55-1,75 $(\mathrm{m}$ ) e 7 (infravermelho médio, 2,08 - 2,35 (m). Cada canal possui resolução espacial de $30 \mathrm{~m}$ e resolução radiométrica de 8 bits (ou 256 níveis de quantização). A resolução temporal da plataforma é de 16 dias. A imagem foi escolhida por apresentar diversos tipos diferentes de cobertura do solo sem cobertura de nuvens, além de estar disponível no servidor do USGS nas versões sem calibração radiométrica e com reflectância de superfície.

A figura 1 exibe a imagem da área de estudo, no sistema de referência WGS84, e sistema de projeção UTM, fuso 21 S.

Figura 1- Imagem Landsat 7 (órbita/ponto 226/o71) da área de estudo, composição B(3)G(4)R(5), sistema de referência WGS84, sistema de projeção

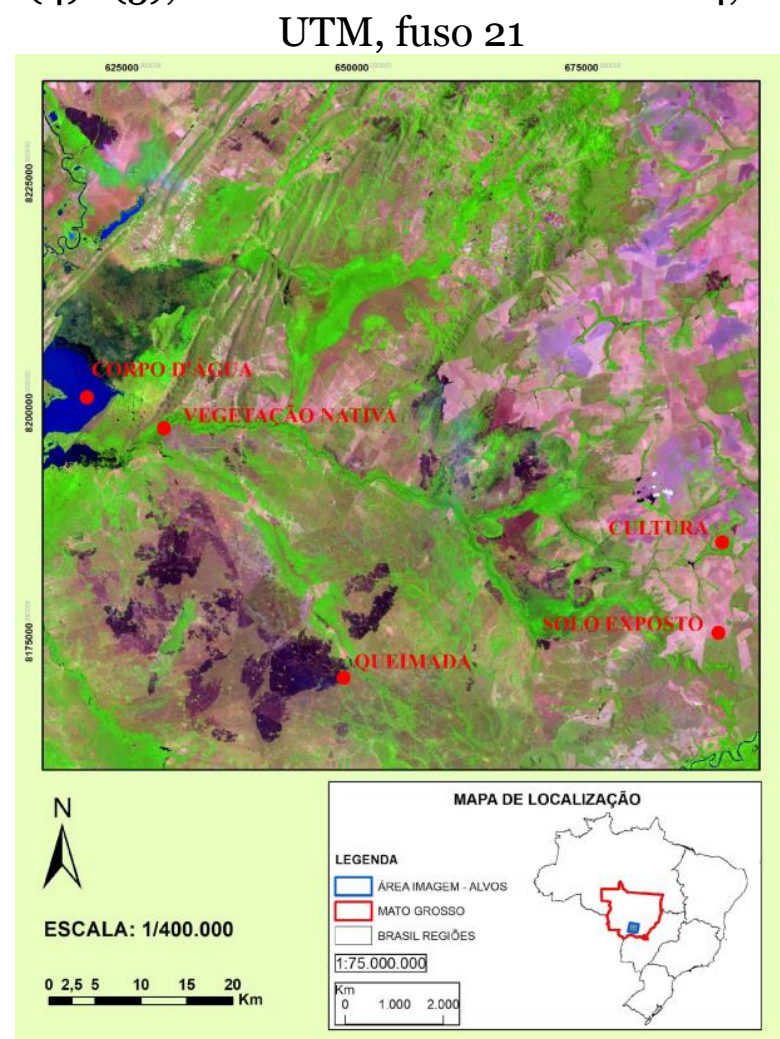

Fonte: Autoria própria 
Na figura 1, estão marcados, com anotações em vermelho, os alvos estudados: área queimada, corpo d'água, cultura agrícola, vegetação nativa e solo exposto. A imagem é exibida na composição B(3)G(4)R(5), para realçar a resposta da vegetação no infravermelho próximo e também áreas queimadas no infravermelho de ondas curtas.

Esta cena, nesta data, foi escolhida por apresentar uma grande diversidade de alvos com características espectrais distintas, tais como áreas de área queimada, corpos d'água, cultura agrícola, vegetação nativa (de grande porte) e solo exposto, bem como uma quantidade muito pequena de nuvens (concentradas no canto superior direito da cena).

\section{MÉTODO}

A figura 2 exibe um fluxograma com cada uma das etapas executadas no presente trabalho.

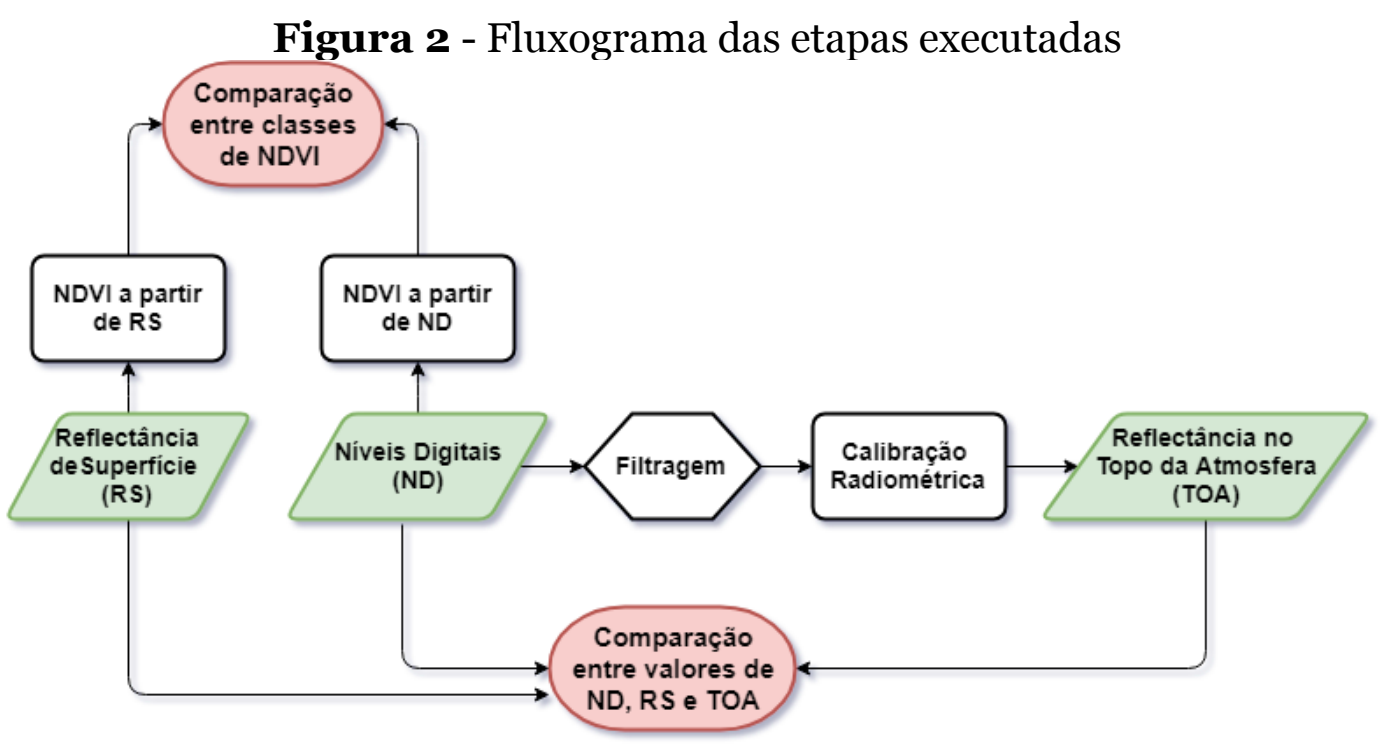

Fonte: Autoria própria

Foram adquiridas duas versões da mesma cena Landsat 7 (sensor ETM+) junto ao Serviço Geológico dos Estados Unidos (USGS, disponível em https://earthexplorer.usgs.gov). Uma delas em unidades de ND (sem calibração radiométrica e sem correção atmosférica) e a outra com correção atmosférica (em unidades de reflectância de superfície, portanto). As imagens Landsat 7 com correção atmosférica são processadas (USGS, 2019a; 2019b) com o 
LEDAPS (Landsat Ecosystem Disturbance Adaptive Processing System). No processo, utiliza-se o código 6S (Second Simulation of the Satellite Signal in the Solar Spectrum) como modelo de transferência radiativa da atmosfera para a conversão dos dados brutos em reflectância de superfície.

A versão bruta da imagem, em unidades de ND (com resolução radiométrica de 8 bits, isto é, com tonalidades de cinza variando dentro do intervalo [0, 255]), foi submetida a um processamento para converter seu contradomínio em unidades de reflectância no topo da atmosfera, no intervalo [0,1]. Previamente, aplicou-se uma filtragem por mediana (máscara 3×3), para eliminar diversos ruídos (erros de bit, inerentes ao próprio sistema sensor ou causados em decorrência de falhas no processo de transmissão dos dados) presentes na cena. Em seguida, aplicaram-se os parâmetros de calibração radiométrica (CHANDER et al., 2009; GÜRTLER et al. 2005) para a conversão de ND em radiância espectral, em unidades de $w m^{-2} s r^{-1} \mu m^{-1}$, empregando-se a equação 1 (USGS, 2019b):

$L_{\lambda}=\left(\frac{L_{\max }-L_{\min }}{255}\right) \cdot N D+L_{\min }$

Em que $L_{\lambda}$ é a radiância espectral $\left(w m^{-2} s r^{-1} \mu m^{-1}\right)$ na banda $\lambda, L_{\max }$ e $L_{\text {min }}$ são, respectivamente, as radiâncias máxima e mínima esperadas para a banda $\lambda$ e $N D$ são os valores da imagem de entrada.

Os valores de radiância, então, foram convertidos em reflectância no topo da atmosfera (TOA) pela seguinte equação (USGS, 2019b; PONZONI et al., 2015):

$\rho_{\lambda}=\frac{\pi \cdot L_{\lambda} \cdot d^{2}}{E \operatorname{SUN} N_{\lambda} \cdot \cos \theta}$

Em que $\rho_{\lambda}$ é a reflectância espectral na banda $\angle$ no topo da atmosfera, $d$ é a distância Terra-Sol média, em unidades astronômicas (UA), $\operatorname{ESUN}_{\lambda}$ é a irradiância solar média (PONZONI et al., 2015; NOVO, 2010) no topo da 
atmosfera (em $\mathrm{wm}^{-2} \mu \mathrm{m}^{-1}$ ) para a banda $/ \mathrm{e}$ (é o ângulo zenital solar no centro da cena no momento da aquisição.

A distância Terra-Sol média $d$ pode ser obtida da seguinte relação (STEIN et al., 2002):

$d=1+0,0167 \cdot \operatorname{sen}\left[\frac{2 \pi \cdot(D D A-93,5)}{365}\right]$.

Em que $D D A$ é o dia do ano, contado a partir do dia 01 de janeiro do ano da tomada da cena.

A partir de então, estavam disponíveis três versões da mesma cena: os dados brutos, em unidades de ND, uma imagem de reflectância no topo da atmosfera (TOA, sem correção atmosférica) e outra imagem de reflectância de superfície (RS, corrigida de efeitos atmosféricos pela aplicação do algoritmo 6S). Para comparação destes três dados, foram selecionadas as seguintes classes de informação: área queimada, corpo d'água, cultura agrícola, vegetação nativa e solo exposto, identificadas a partir de interpretação visual de composições coloridas das cenas.

Amostras com área de 9 pixels $(3 \times 3)$ de cada uma das classes foram coletadas, e seus valores médios foram calculados nas bandas 1, 2, 3, 4, 5 e 7 . Optou-se por distribuições pequenas (9 pixels) para se evitar que a variância das amostras dentro de cada retângulo fosse grande. Além disso, a média garante que erros de registro entre as bandas não interfiram nas análises posteriores (se apenas o valor de um pixel fosse coletado, não seria possível assegurar que ele representasse exatamente a mesma área de 30 x $30 \mathrm{~m}$ no terreno em todas as bandas).

Em seguida, foram calculados índices de vegetação NDVI para os dados de reflectância de superfície e para os dados em níveis digitais, conforme a equação 4 (JENSEN, 2009):

$N D V I=\frac{\rho_{i v p}-\rho_{v}}{\rho_{i v p}+\rho_{v}}$ 
Em que $\rho_{i v p}$ é a reflectância no infravermelho próximo e $\rho_{v}$ é a reflectância no vermelho.

Como se percebe pela equação 4, o NDVI é calculado a partir das reflectâncias nas bandas do vermelho e infravermelho próximo. A razão de bandas utilizando dados brutos de ND não é, em rigor, um índice de vegetação. Isso porque, ao não se fazer a calibração radiométrica dos dados, negligencia-se a função de transferência dos CCD, que é particular para cada uma das bandas (ganhos e offsets diferentes). Entretanto, como vários autores assim o fazem, tal razão de bandas será também chamada neste trabalho de NDVI, apenas por simplicidade. Os efeitos desse erro (isto é, a não adoção de reflectâncias na equação 4) serão analisados no próximo item de resultados, quando serão comparados os índices formados a partir de RS e ND.

\section{RESULTADOS E DISCUSSÃo}

A seguir, no item 5.1, são apresentadas e discutidas as assinaturas espectrais obtidas para cada alvo de interesse ao longo da resolução espectral do Landsat 7, calculadas para valores de ND, TOA e RS. No item 5.2, apresentamse os NDVI calculados para valores de ND e RS.

\section{Comparação entre curvas espectrais}

As curvas espectrais dos alvos área queimada, corpo d’água, cultura agrícola, vegetação nativa e solo exposto (marcados na figura 1) foram obtidas a partir da interpretação das cenas e extração de valores de pixels (médias de uma região de $3 \times 3$ pixels centrada em cada classe de informação) em todas as 7 bandas do sensor. Para cada alvo, foram construídas três curvas com base em seus valores nas imagens bruta (ND), de reflectância no topo da atmosfera (TOA) e reflectância de superfície (RS). Para facilitar a visualização dos valores, as quantidades de ND foram normalizadas para o intervalo [0,1], de maneira que pudessem ser exibidos em mesma escala que os dados de reflectância nos gráficos.

A figura 3 mostra os valores para o alvo queimada. Este alvo tem como característica espectral baixos valores nas bandas do visível (bandas 1-3) e 
infravermelho próximo (banda 4). Da análise desta figura percebe-se que a curva de ND é completamente diferente as curvas de reflectância, e não condiz com o comportamento esperado. Ao analisar as curvas de reflectância para queimada, é possível perceber o efeito da atmosfera na curva de TOA, fazendo com que as bandas apresentem valores mais elevados que aqueles da RS, especialmente na banda do azul, em que o espalhamento atmosférico é maior.

Figura 3 - Gráfico dos valores de ND, TOA e RS por banda do alvo queimada. Os valores de ND foram normalizados para o intervalo [0, 1] para melhor visualização

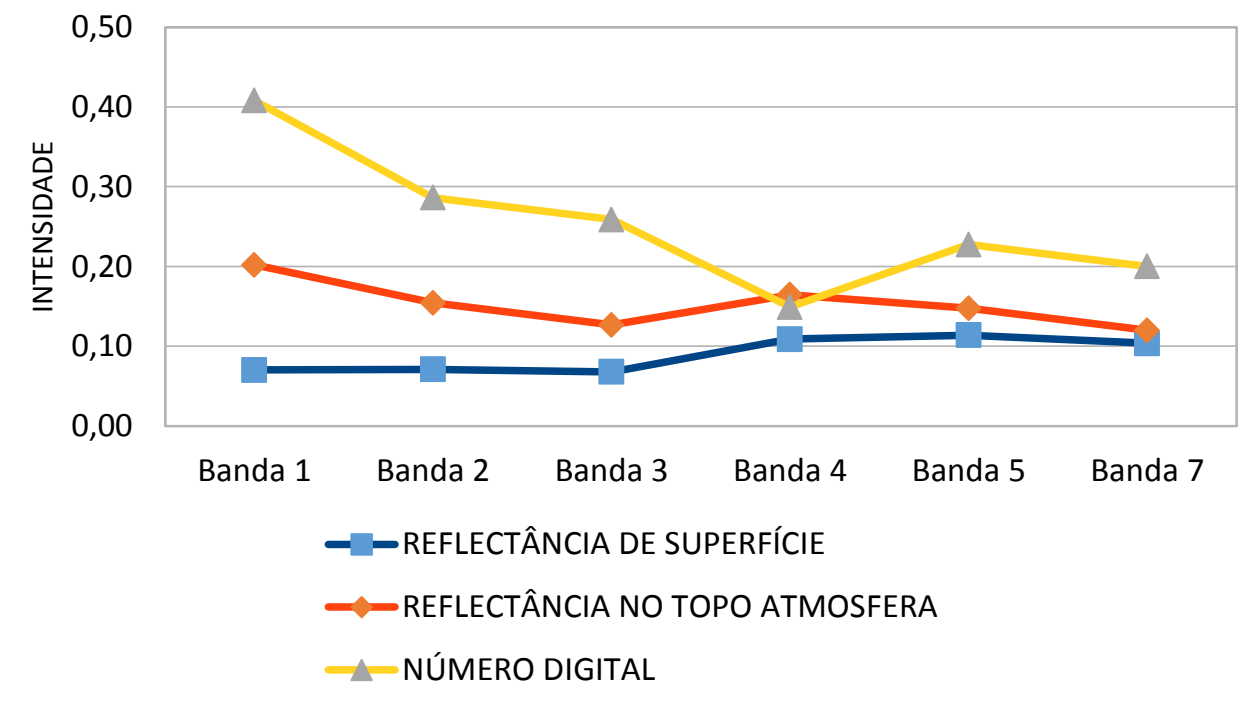

Fonte: Autoria própria

A figura 4 exibe os valores obtidos para o alvo corpo d'água sem sedimentos suspensos. 
Figura 4 - Gráfico dos valores de ND, TOA e RS por banda do alvo corpo d'água. Os valores de ND foram normalizados para o intervalo $[0,1]$ para melhor visualização

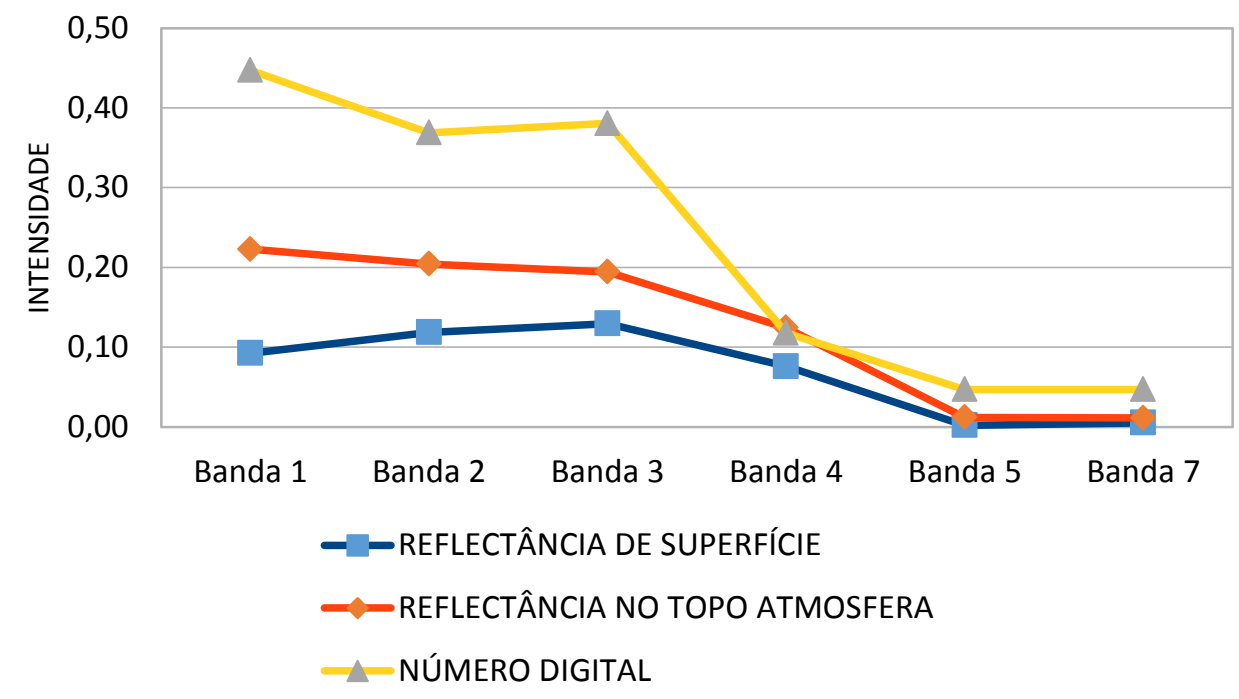

Fonte: Autoria própria

O comportamento que mais destoa entre as três curvas na figura 4 está em ND, principalmente nas bandas 1,2 e 3 (bandas do visível), com comportamento parecido a partir da banda 4 até a banda 7 (bandas do infravermelho próximo e médio). O comportamento esperado deste tipo de alvo pode ser visto na figura 5 , a qual exibe o comportamento espectral de alguns tipos de alvos.

Figura 5 - Comportamento espectral esperado dos alvos Grama, Concreto, Solo Arenoso, Telhados, Solo Exposto, Asfalto, Grama Artificial e Água Limpa. Fonte:

JENSEN (2009)

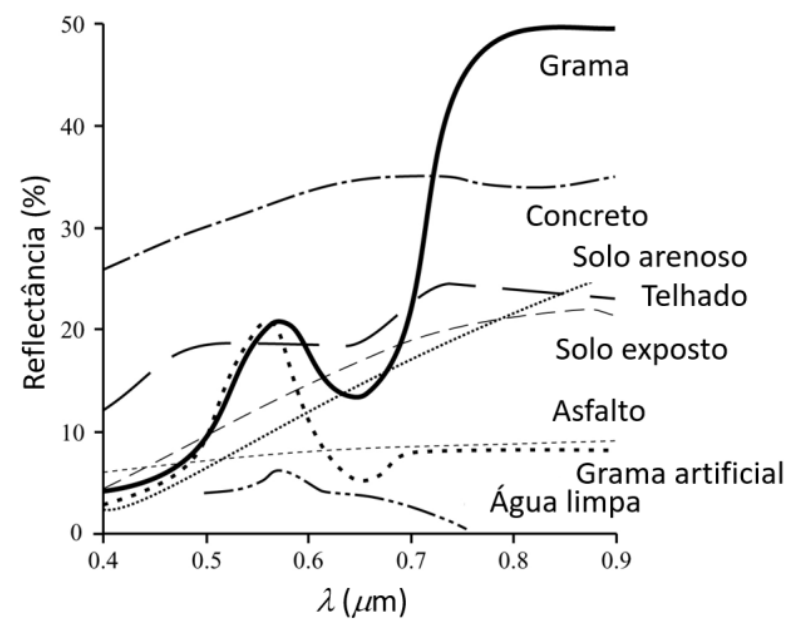

Fonte: Modificado de Jensen (2009) 
Na figura 5, percebe-se que a reflectância da água é muito baixa, se comparada com outros alvos, e, por isso, ela tende a se apresentar bastante escura nas imagens de satélite. Sua reflectância é nula nas bandas do infravermelho, devido à absorção da radiação eletromagnética nestes comprimentos de onda. A água sem sedimentos suspensos apresenta maior reflectância na região espectral do azul, por causa do espalhamento molecular. $\mathrm{Na}$ figura 4, ao se comparar as curvas de TOA e RS percebe-se que o espalhamento atmosférico provoca uma reflectância maior em todas as bandas, mas mais acentuadamente nos menores comprimentos de onda. Além disso, a forma da curva composta por valores de ND é completamente descaracterizada por causa da falta de calibração radiométrica dos dados e correção atmosférica.

Nas figuras 6 e 7 exibem-se as curvas obtidas para os alvos vegetação nativa (de grande porte) e cultura agrícola. As características das curvas de TOA e RS são parecidas no infravermelho, destoando mais no visível. Contudo, é evidente a interferência atmosférica nos dados de reflectância TOA, assim como, do ganho do sensor para o caso do ND. O aspecto da curva obtida com os dados de ND é bastante diferente das curvas de SR e TOA. Nos valores absolutos das curvas de SR e TOA, nas bandas 1, 2 e 3 (visível), observa-se que só na reflectância de superfície (RS) o comportamento está de acordo com o esperado para vegetação (conforme figura 5), ou seja, valores baixos nas bandas do azul e vermelho, e maiores na banda do verde. Isso deve ocorrer para a vegetação sadia, que absorve energia nas faixas do azul e vermelho para fotossíntese, e reflete mais no verde. Alterações neste comportamento são indícios de estresse da planta (causado, por exemplo, pela falta de irrigação, de nutrientes, por pragas, etc.). Percebe-se que efeitos atmosféricos alteram a assinatura esperada para a vegetação, especialmente na banda do azul. Uma análise desta curva levaria a conclusões erradas sobre o estado da vegetação, portanto. 
Figura 6 - Gráfico dos valores de ND, TOA e RS por banda do alvo cultura agrícola. Os valores de ND foram normalizados para o intervalo $[0,1]$ para melhor visualização

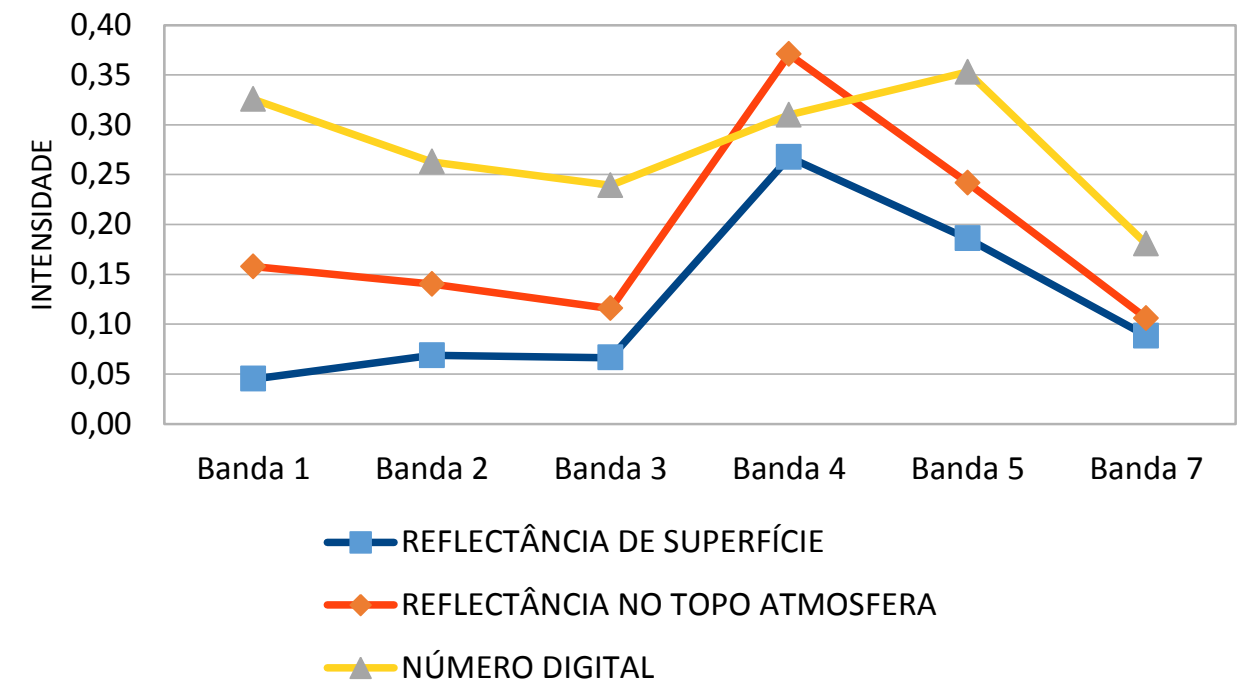

Fonte: Autoria própria

Figura 7 - Gráfico dos valores de ND, TOA e RS por banda do alvo vegetação nativa. Os valores de ND foram normalizados para o intervalo $[0,1]$ para melhor visualização

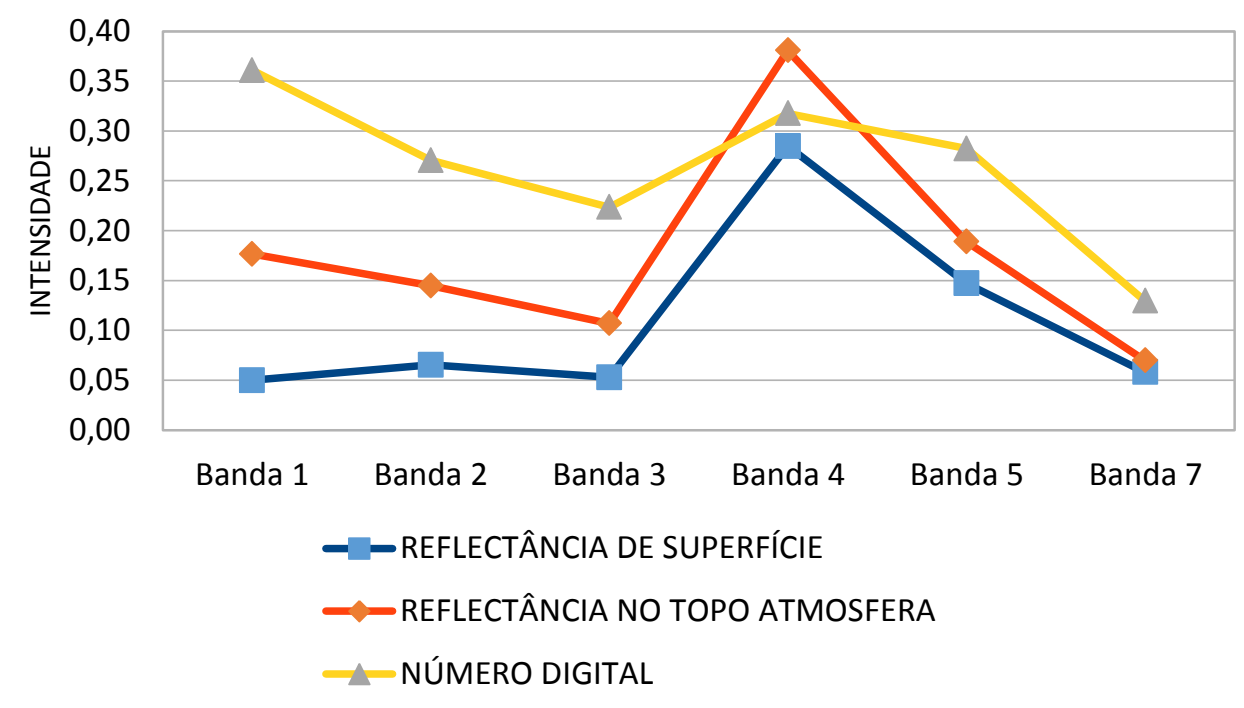

Fonte: Autoria própria

No caso do alvo solo exposto, na figura 8, mais uma vez, a curva de ND difere completamente do comportamento esperado, caracterizado pelas curvas de reflectância (conforme figura 5). A presença de efeitos atmosféricos fica evidente na comparação das curvas de TOA e RS. Na curva de TOA os valores de reflectância são maiores em todas as bandas. 
Figura 8 - Gráfico dos valores de ND, TOA e RS por banda do alvo solo exposto. Os valores de ND foram normalizados para o intervalo $[0,1]$ para melhor visualização

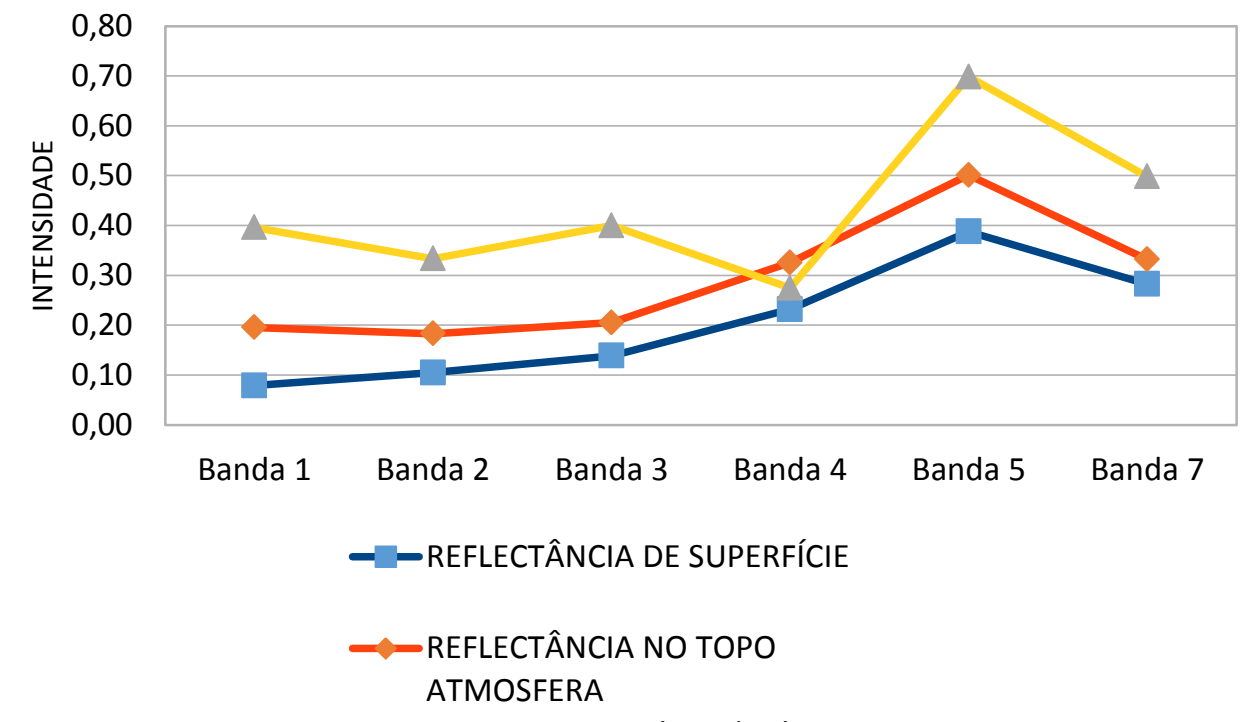

Fonte: Autoria própria

\section{Índice de Vegetação por Diferença Normalizada - NDVI}

A fim de analisar qualitativamente e quantitativamente o impacto da falta de calibração radiométrica e correção atmosférica nas informações extraídas de imagens de sensoriamento remoto, foram calculados NDVI a partir da imagem de ND e da imagem de RS. Em consonância com as comparações feitas nas figuras (3 a 8), em que todos os alvos sem correção se comportaram de maneira diferente em relação às assinaturas espectrais esperadas (nos dados corrigidos), nota-se grande discrepância entre os NDVI.

Os mapas temáticos gerados a partir dos índices são exibidos na figura 9. A paleta de cores e as classes dos mapas foram configuradas para ser coincidentes. 
Figura 9 - Mapas temáticos gerados a partir dos NDVI calculados com valores de ND (A) e RS (B). As classes temáticas foram definidas de maneira idêntica para ambos, conforme a legenda

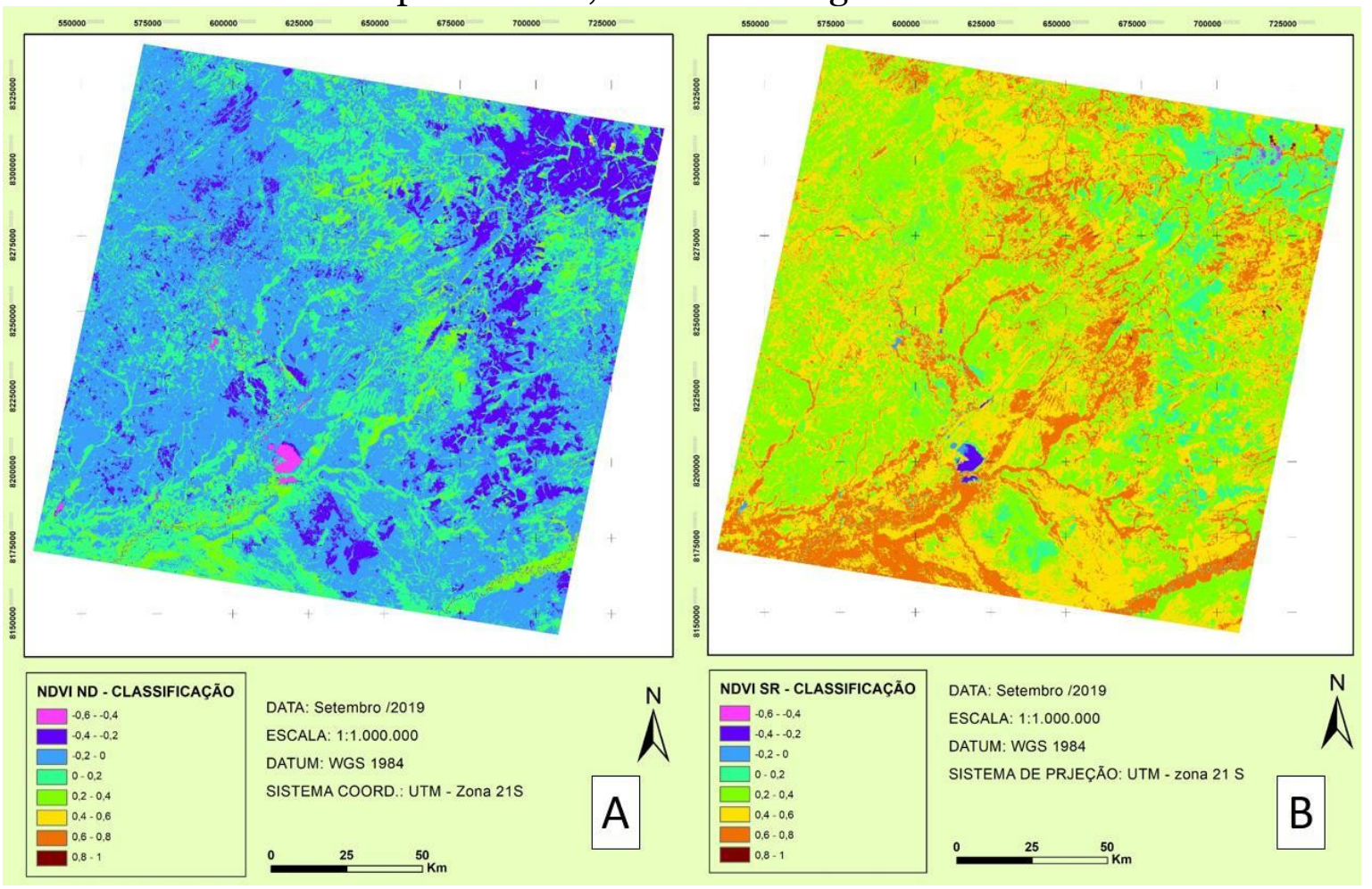

Fonte: Autoria própria

Os valores de um NDVI ocupam o intervalo [-1,1]. Nestes índices, pixels de vegetação sadia apresentam valores próximos de 1. Solo exposto, geralmente, apresenta valores positivos baixos, entre 0,3 e o. Valores negativos são produzidos por sombra e água. O intervalo de valores predominantes no NDVI da imagem de ND (figura 9A) é de $[-0,4,-0,2]$, que corresponderia a pixels de sombra ou água, enquanto na imagem com correção atmosférica (RS) o intervalo predominante (figura 9B) é de $[0,2,0,4]$, correspondendo a solo exposto. Existe, portanto, significativa diferença para os resultados de NDVI. Esta diferença é causada pela interferência incontestável da atmosfera na reflectância em produtos gerados por sensoriamento remoto orbital, além da falta de calibração radiométrica.

A seguir, nas figuras 10 até 14 são exibidos os alvos analisados em composição colorida B(3)G(4)R(5), e os NDVI calculados a partir dos dados de ND e RS. Estes estão representados em mapas temáticos, para os quais uma mesma legenda de classes foi empregada. De maneira geral, os NDVI são 
bastante diferentes para os dados brutos e corrigidos, provando que análises feitas sobre razões de bandas calculadas a partir de dados brutos (erroneamente chamados de NDVI) conduzem a conclusões erradas sobre o comportamento dos alvos estudados.

Figura 10 - Classe de informação: queimada. Composição colorida B(3)G(4)R(5) em (A), NDVI a partir dos dados brutos em (B) e NDVI a partir da reflectância de superfície em (C)

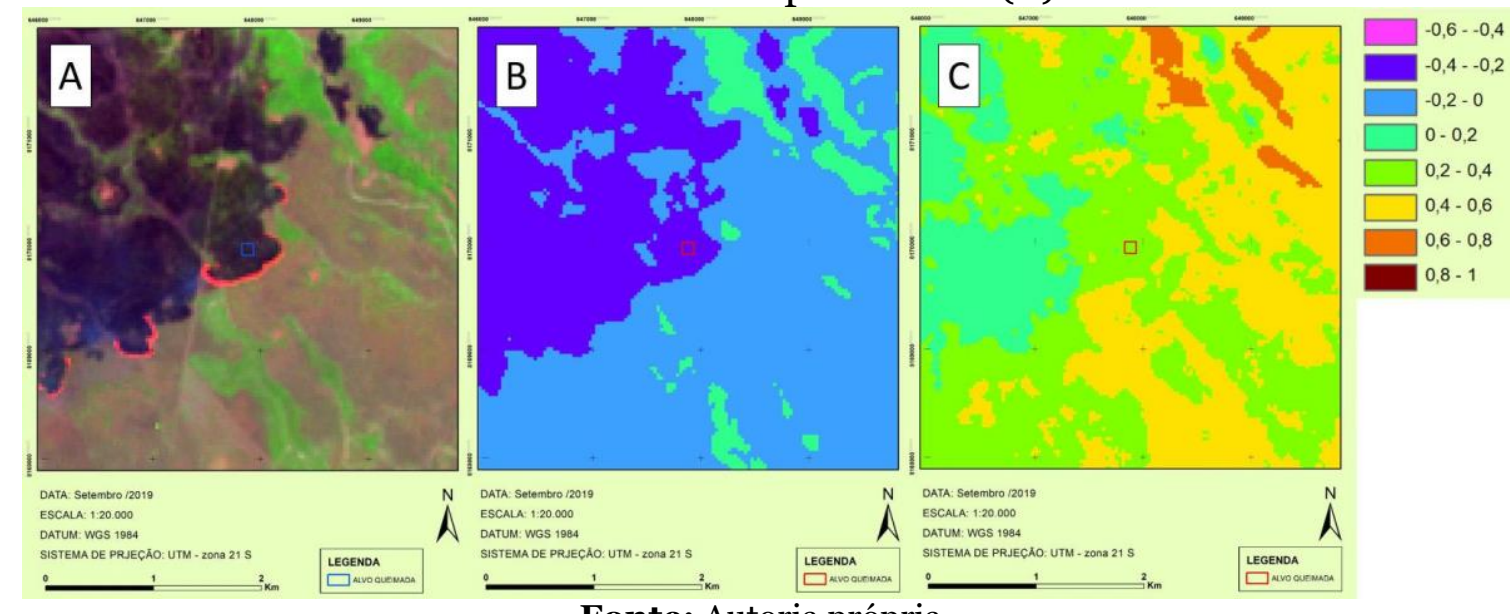

Fonte: Autoria própria

Figura 11 - Classe de informação: corpo d'água. Composição colorida B(3)G(4)R(5) em (A), NDVI a partir dos dados brutos em (B) e NDVI a partir da reflectância de superfície em (C)
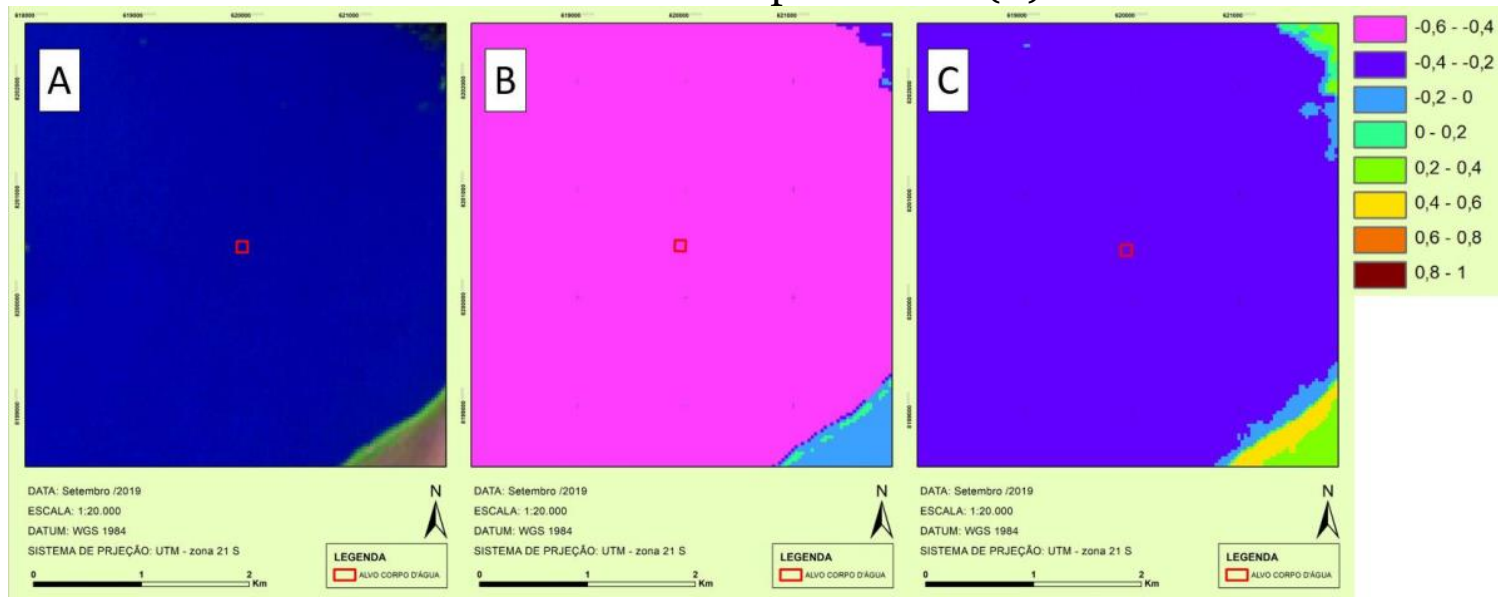

Fonte: Autoria própria 
Figura 12 - Classe de informação: cultura agrícola. Composição colorida $\mathrm{B}(3) \mathrm{G}(4) \mathrm{R}(5)$ em (A), NDVI a partir dos dados brutos em (B) e NDVI a partir da reflectância de superfície em $(\mathrm{C})$

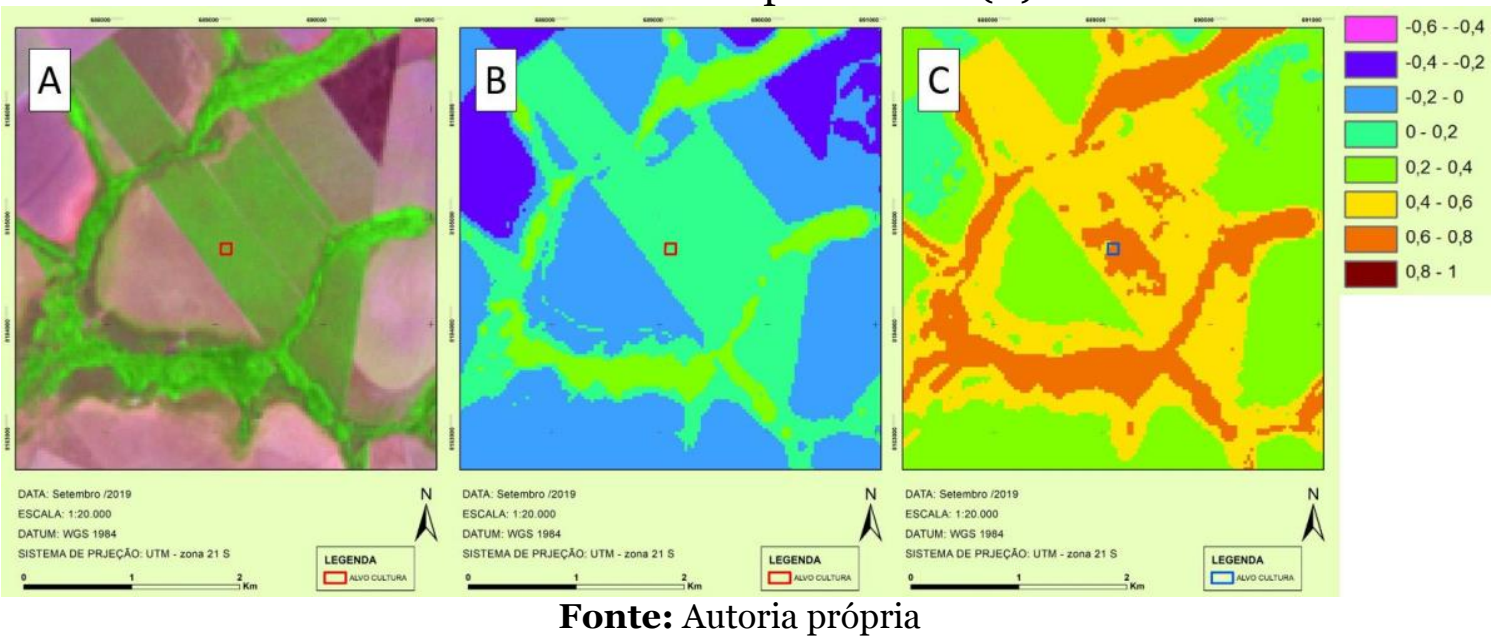

Figura 13 - Classe de informação: vegetação nativa. Composição colorida B(3)G(4)R(5) em (A), NDVI a partir dos dados brutos em (B) e NDVI a partir da reflectância de superfície em (C)

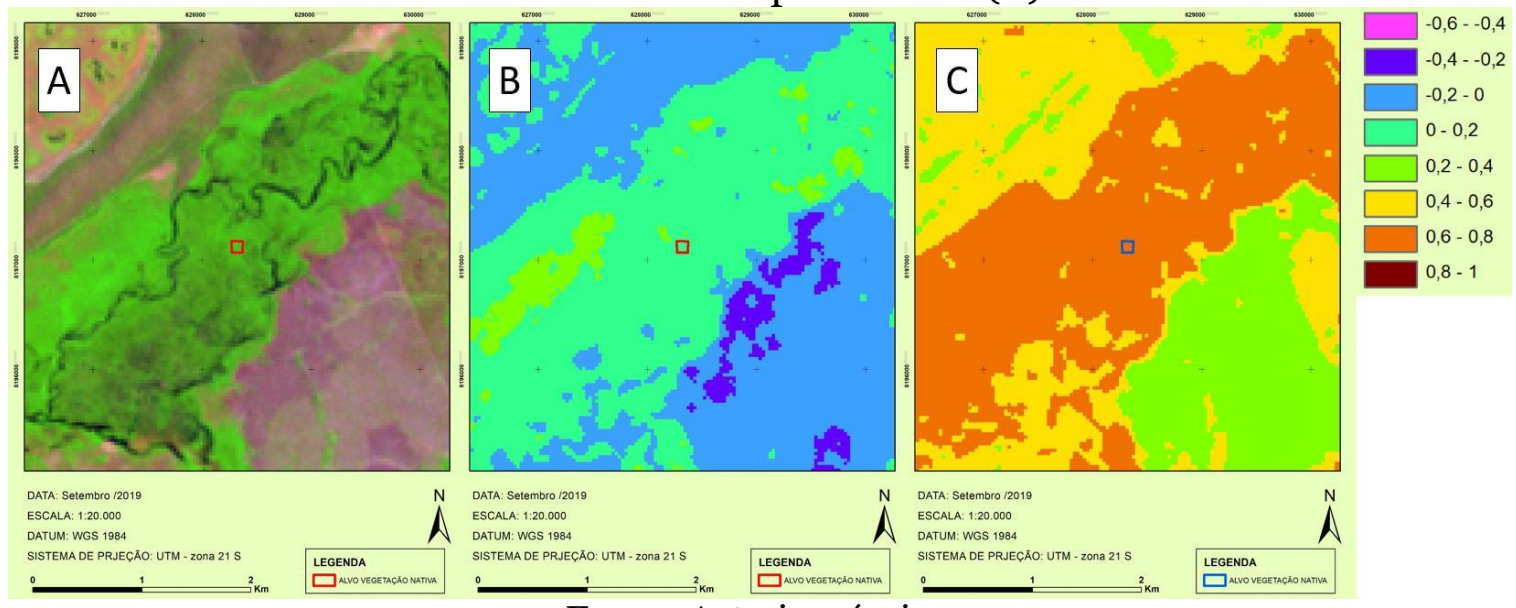

Fonte: Autoria própria 
Figura 14 - Classe de informação: solo exposto. Composição colorida B(3)G(4)R(5) em (A), NDVI a partir dos dados brutos em (B) e NDVI a partir da reflectância de superfície em (C)

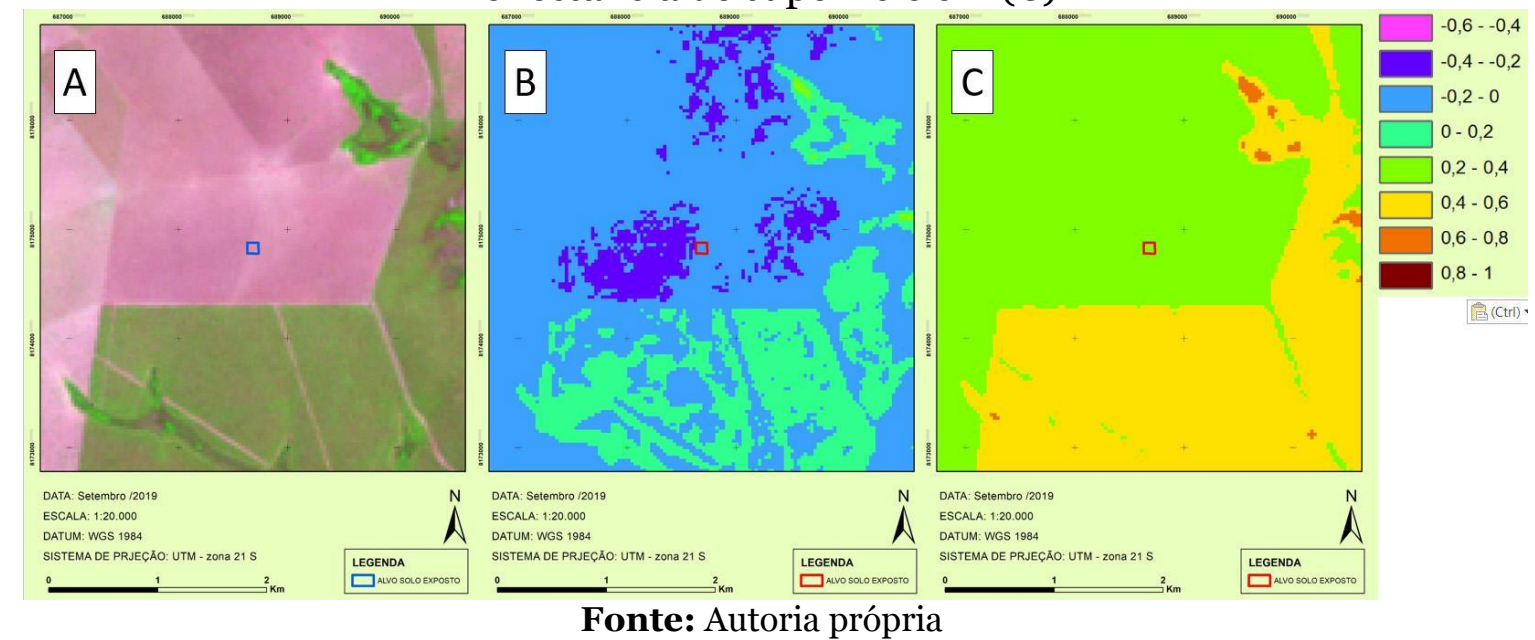

Da análise das figuras 10 a 14 fica evidente que a prática comum de se utilizarem os dados de ND diretamente na equação 4 produz resultados enviesados. As classes de informação são completamente descaracterizadas nos NDVI calculados dos valores de ND. Informações extraídas de tais produtos estarão comprometidas e a análise destas informações levará a conclusões erradas sobre o comportamento dos alvos apresentados.

\section{CONSIDERAÇÕES FINAIS}

Os resultados obtidos demonstram a importância incontestável de se aplicar a calibração radiométrica e correção atmosférica para análises de imagens de satélite. Nos gráficos em que se apresentam os valores de reflectância de superfície, reflectância no topo da atmosfera e número digital das bandas 1, 2, 3, 4, 5 e 7, para os alvos área queimada, cultura agrícola, vegetação nativa, copo d'água e solo exposto é visível o comportamento de curva diferente e valores absolutos diferentes para o mesmo pixel, nos diferentes produtos. Obviamente, valores de ND [0, 255] serão largamente diferentes dos valores de reflectância $[0,1]$ por estarem em escalas diferentes, mas, para além disso, as formas das assinaturas espectrais dos alvos também são amplamente diferentes. De fato, a assinatura esperada para os alvos é completamente descaracterizada quando são empregados valores de ND. 
Somados à falta de calibração radiométrica, os efeitos atmosféricos alteram as curvas de reflectância, como demonstrado. Trabalhos que utilizem os valores de reflectância em suas análises podem ser comprometidos e não representar fidedignamente a realidade em campo, caso não levem em consideração as interferências causadas pela atmosfera, como absorção e espalhamento. Foi demonstrado que, apenas quando foram utilizados dados de reflectância de superfície, obtidas pela utilização do algoritmo 6S, as curvas de reflectância espectral dos alvos foram coincidentes com as esperadas.

Por fim, na geração de mapas temáticos, a partir do cálculo de NDVI dos dados de ND e RS, observa-se grande diferença nos intervalos de classificação, que geram um aspecto visual diferente para os índices baseados em reflectância de superfície e número digital. Mais uma evidência, portanto, da necessidade de se aplicarem a calibração radiométrica e a correção de efeitos atmosféricos aos dados de sensoriamento remoto orbital antes que estes possam ser analisados.

\section{REFERÊNCIAS}

ANDRADE, C. B.; OLIVEIRA, L. M. M.; OMENA, J. A. M.; VILLAR A. C.; GUSMÃO, L.; RODRIGUES, D. F. B. Avaliação de índices de vegetação e características fisiográficas no Sertão Pernambucano. Revista Brasileira de Meio Ambiente, v.4, n.1. p. 97-107, 2018.

ANTUNES, H. M. A.; FREIRE, B. M. R.; BOTELHO, S. A.; TONIOLLI H. L. Correções Atmosféricas de Imagens de Satélites Utilizando o Modelo 6S. In: XXI Congresso Brasileiro de Cartografia, Belo Horizonte. Anais do Congresso Brasileiro de Cartografia, 2003. p. 1-6.

CHANDER, G. C.; MARKHAM, B. L.; HELDER, D. L. Summary of Current radiometric calibration coefficients for Landsat MSS, TM, ETM+ and EO-1 ALI sensors. Remote Sensing of Environment, v. 113, n. 5, 2009.

CHAVEZ, P. S. An improved dark-object subtraction technique for atmospheric scattering correction of multispectral data. Remote sensing of environment, 24(3), 459-479, 1988.

GÜRTLER, S.; EPIPHANIO, J. C. N.; LUIZ, A. J. B.; FORMAGGIO, A. R. Planilha Eletrônica para o Cálculo da Reflectância em Imagens TM e ETM+ Landsat. Revista Brasileira de Cartografia, v. 2, n. 57, 2005. 
JENSEN, J. Sensoriamento e remoto do ambiente: uma perspectiva em recursos terrestres. Tradução, prefácio e notas: Jose Carlos Neves Epiphanio (coordenador).São Jose dos Campos - SP: Parêntese, 2009.

LEITE, A. P.; SANTOS, G. R.; SANTOS, J. E. O. Análise temporal dos índices de vegetação NDVI e SAVI na estação experimental de Itatinga utilizando imagens Landsat 8. Revista Brasileira de Energias Renováveis. Vol. 6, n. 4, p. 606-623, 2017.

MATTHEW, M. W.; ADLER-GOLDEN, S. M.; BERK, A.; FELDE, G.; ANDERSON, G. P.; GORODETZKY, D.; SHIPPERT, M. Atmospheric correction of spectral imagery: evaluation of the FLAASH algorithm with AVIRIS data. In Applied Imagery Pattern Recognition Workshop, 2002. Proceedings. (pp. 157-163). IEEE.

NOVO, E. M. L. M. Sensoriamento remoto: princípios e aplicações. São Paulo: Blucher, 2010.

PONZONI, F. J.; PINTO, C. T.; LAMPARELLI, R. A. C.; JUNIOR, J. Z.; ANTUNES, M. A. H. Calibração de Sensores Orbitais. São Paulo: Oficina de Textos, 2015 .

RIBEIRO, B. M. R.; PETRY, F. A.; LIMBERGER, A. R. Análise Temporal de dados NDVI para o município de Toledo PR, obtidos de imagens Landsat 8. Revista Cultivando o Saber, vol. 11, n. 2, p. 149-159, Abr/Jun. 2018.

RUMORA, L.; MILER, M.; MEDAK, D. Impact of Various Atmospheric Corrections on Sentinel-2 Land Cover Classification Accuracy Using Machine Learning Classifiers. ISPRS International Journal of Geo-Information, 9(4), 277, 2020.

SILVIA, A. M.; MOREIRA, E. B. M. Avaliação espaço-temporal do índice de vegetação (NDVI) no manguezal de Maracaípe - PE, através de imagens do satélite Landsat 5 TM. In: XV Simpósio Brasileiro de Sensoriamento Remoto, Curitiba. Anais do Simpósio Brasileiro de Sensoriamento Remoto, 2011. p. 1570-1575.

STEIN, A.; MEER, F. D.; GORTE, B. Spatial Statistics for Remote Sensing. Springer, 2002.

USGS - United States Geological Survey. LANDSAT 4-7 Surface

Reflectance (LEDAPS) Product Guide, version 2. Relatório Técnico. 2019a.

USGS - United States Geological Survey. LANDSAT 7 Data Users

Handbook, version 2. Relatório Técnico. 2019b.

VELASCO, G. N.; POLIZEL, J. L.; COLTRI, P. P.; LIMA, A. M. L. P.; FILHO, D. F. S. Aplicação do Índice de Vegetação NDVI (Normalized Diference Vegetation 
Index) em Imagens de Alta Resolução no Município de São Paulo e suas Limitações. Revista da Sociedade Brasileira de Arborização Urbana, vol. 2, n. 3, 2007.

VERMOTE, E. F.; TANRE, D.; DEUZE, J. L.; HERMAN, M. \& MORCRETTE, J. J. Second Simulation of the Satellite Signal in the Solar Spectrum, 6S: an overview. IEEE Trans. Geosc. and Remote Sens. vol. 35, n. 3, p. 675- 686, 1997.

ZANZARINI, F. V.; PISSARRA, T. C. T.; BRANDÃO, F. J. C.; TEIXEIRA, D. D. B. Correlação Espacial do índice de vegetação (NDVI) de imagem Landsat/ETM+ com atributos do solo. Revista Brasileira de Engenharia Agrícola e Ambiental, vol. 17, n. 6, p. 608-614, 2013.

Recebido em 12 de Agosto de 2020

Aceito em 16 de Dezembro de 2020 